\title{
Entrevista Educativa
}

Formato y Manual de Entrevista

Garzón Moreno Ana María

Nieves Cruz Gabriela

Sperandío Contreras Ana Gabriela

Vargas Atencia María Isabel

\section{Docente}

PS. Ma. Lina María Pacheco Palencia

Facultad de Psicología

Villavicencio

2020 


\section{Entrevista Educativa Formato}

\section{Universidad Santo Tomás}

\section{Lugar donde se llevará a cabo la entrevista:}

\section{Fecha:}

Condiciones éticas: El siguiente formato de entrevista se realizará y ejecutará en un ámbito escolar, por consiguiente, es de gran importancia pedir el consentimiento informado del padre y del alumno y el permiso de la institución para que este proceso se pueda llevar a cabo, en caso de que el estudiante sea menor de edad, se estará pidiendo un asentimiento informado.

También se le explicará al estudiante sobre la confidencialidad de sus datos personales y de la información que nos dé (aclarandole al estudiante que la confidencialidad de la información que él no quiera que se comparta no serán revelada; sin embargo, hay que tenerse en cuenta que dado al proceso evaluativo y las condiciones institucionales de evaluación y seguimiento, se elaborarán informes con las generalidades que se relacionen con el motivo de la entrevista)

El entrevistador, tiene también el deber de ser respetuoso en todo momento con la persona a la que va a entrevistar, tomando en cuenta las siguientes condiciones: No se le alzara la voz en ningún momento al entrevistado cuando se esté explicando el procedimiento, le expondrá los resultados que obtenga al finalizar la entrevista y será claro, va a aceptar si la persona ya no quiere continuar con la entrevista, va a tomar una postura objetiva sin usar sus sesgos personales.

Objetivo de la entrevista: Evaluar mediante una entrevista semi-estructurada las habilidades sociales que tienen los estudiantes dentro del aula de clase, y cómo esto facilita su convivencia; ya que se han reportado alumnos que interfieren y hacen imposible esto. A su vez, se busca discutir las declaraciones realizadas a sus conductas.

Tipo de entrevista según su variación: Se utilizará la entrevista narrativa ¿Por qué? Por el hecho de que se pretende conocer el cómo y el porqué de las conductas que ha venido desarrollando el estudiante.

En siguiente formato de asentimiento informado,es una adaptación al obtenido del Comité de ética, bioética e integridad científica, de la Universidad Santo Tomás sede Villavicencio. 


\section{Asentimiento informado}

Fecha: LI- CÓD:

En el marco de la Constitución Política Nacional de Colombia, la Ley 1098 de 2006 Código de la Infancia y la Adolescencia y demás normatividad aplicable vigente, y del acuerdo institucional de la Universidad Santo Tomás No. 32 del 3 de julio de 2019, en el artículo 4, principios 2 (responsabilidad) y 6 (integridad); y, considerando las características de la investigación, se requiere que usted lea detenidamente y si está de acuerdo con su contenido, exprese su permiso o nó, firmando el siguiente documento:

Respetado(a)

Le invitamos a vincularse como participante al proceso de entrevista, el cual ha sido aprobado por su padre o tutor responsable; el cual será llevado a cabo por las psicólogas educativas Ana María Garzón, Gabriela Nieves, Ana Sperandío y Maria Isabel Vargas; adscritas a la unidad de UDIES

El objetivo de este estudio es:

Evaluar mediante una entrevista semi-estructurada las habilidades sociales que tienen los estudiantes dentro del aula de clase, y cómo esto facilita su convivencia; ya que se han reportado alumnos que interfieren y hacen imposible esto. A su vez, se busca discutir las declaraciones realizadas a sus conductas.

Y este estudio se realiza porque:

Las habilidades sociales (como herramienta para una buena convivencia) ayudan a expresar sentimientos, actitudes, deseos y derechos de manera adecuada y efectiva; además en el ámbito escolar estas habilidades ayudan a un adecuado ajuste personal, mejores logros escolares y adaptación social. Por ende, las habilidades sociales son tomadas como el tema de evaluación principal en el proceso de la entrevista, con el fin, que de acuerdo a los resultados obtenidos se pasará a elaborar una estrategia pedagógica beneficiosa para los entrevistados, para afianzar más estas habilidades, y así poder generar una posible mejora en su convivencia. 
El procedimiento es: diligenciamiento del consentimiento informado por parte del tutor (si el estudiante es mayor de edad no necesita el permiso de los padres), asentimiento informado por parte del estudiante siendo menor de edad o consentimiento si es mayor de edad. Se hará un proceso de entrevista para identificar cuáles son sus habilidades sociales, posteriormente a esto, se le indicará los resultados obtenidos de este proceso y se le formularán los pasos a seguir.

\section{Tenga en cuenta lo siguiente:}

La participación es voluntaria con las siguientes consideraciones:

1. La investigación se acoge a Art 15 de la constitución política de Colombia, que expide la ley 1581 del 2012 "sistema de protección de datos": Los datos suministrados por los participantes, serán protegidos en todas las fases de la investigación. Por lo tanto, estos datos no serán divulgados, por ningún medio y serán usado únicamente para fines de la presente investigación, datos que serán importantes para que la investigación tenga validez en el territorio a que se destina.

2. La evaluación a partir de la entrevista será regido por el Código Deontológico y Bioético del psicólogo y los estándares de la Ley 1090 del 2006

3. De acuerdo a la Resolución 8430 de 1993 "Por la cual se establecen las normas científicas, técnicas y administrativas para la investigación en salud"; y que el Comité de Ética, Bioética e Integridad Científica de la Universidad Santo Tomás acoge en los demás campos, se establece que

- La participación en la investigación no representa ningún riesgo, algún daño como consecuencia inmediata o tardía del estudio.

- Se entiende por asentimiento informado el acuerdo por escrito, mediante el cual el sujeto de investigación o en su caso, autoriza su participación en la investigación, con pleno conocimiento de la naturaleza de los procedimientos, beneficios y riesgos a que se someterá, con la capacidad de libre elección y sin coacción alguna.

- El participante tiene la libertad de retirar su consentimiento en cualquier momento y dejar de participar en el estudio sin que por ello se creen perjuicios

- La identidad y privacidad de/los participantes no será divulgada 
- Es compromiso del profesional e investigador, proporcionarle informaciónactualizada obtenida durante el estudio, aunque ésta pudiera afectar la voluntad del sujeto para continuar participando.

\section{CONTACTO}

Si desea obtener mayor información sobre el proceso y la investigación puede comunicarse a través de los siguientes correos electrónicos institucionales: anasperandio@usantotomas.edu.co gabrielanieves@usantotomas.edu.co

\section{$\underline{\text { Una vez leído el consentimiento }}$}

Yo identidad No/NUIP $\mathrm{N}^{\circ}$ de

$\mathrm{N}^{\circ} \quad$ de celular: identificado con Registro civil/ tarjeta de de , con domicilio en laciudad Dirección: Teléfono y Correo electrónico: Código del participante;

\section{Declaro que:}

1. He sido invitado(a) a participar en el estudio o investigación de manera voluntaria.

2. He leído y entendido este formato de consentimiento informado o el mismo se me ha leído y explicado.

3. Todas mis preguntas han sido contestadas claramente y he tenido el tiempo suficiente para pensar acerca de mi decisión de participar.

4. He sido informado y conozco de forma detallada los posibles riesgos y beneficios derivados de mi participación en el proyecto.

5. No tengo ninguna duda sobre mi participación, por lo que estoy de acuerdo en hacer parte de esta investigación.

6. Puedo dejar de participar en cualquier momento sin que esto tenga consecuencias.

7.Conozco el mecanismo mediante el cual los investigadores garantizan la custodia y confidencialidad de mis datos, los cuales no serán publicados ni revelados a menos que autorice por escrito lo contrario.

8. Identificó que los investigadores podrían hacer uso de la información y las grabaciones de audio, video o imágenes que se generen en el marco del proyecto. 
9. Sobre esta investigación me asisten los derechos de acceso, rectificación y oposición que podré ejercer mediante solicitud ante el investigador responsable, en la dirección de contacto que figura en este documento.

\begin{tabular}{|l|l|l|l|}
\hline Acepto Participar & $\begin{array}{l}\text { No acepto } \\
\text { participar }\end{array}$ & \\
& & \\
\hline
\end{tabular}

Por tanto: Podrá marcar con una "X". En caso de que acepte participar lograra continuar con el proceso entrevista, por lo contrario, si no acepta no seguirá.

Nombre del niño, niña o adolescente:

$\mathrm{N}^{\mathrm{o}}$ Identificación:

Firma del adulto responsable del menor:

Nombre del adulto responsable del menor:

$\mathrm{N}^{\circ}$ de identificación:

Teléfono:

Correo electrónico:

\section{Declaración del (los) Investigador (es):}

Yo certifico que le he explicado al adulto responsable del niño o adolescente la naturaleza y el objeto de la presente investigación y los posibles riesgos y beneficios que puedan surgir de la misma. Adicionalmente, le he absuelto ampliamente las dudas que ha planteado y le he explicado con precisión el contenido del presente formato de consentimiento informado. Dejo constancia que en todo momento el respeto de los derechos el menor o el adolescente serán prioridad y se acogerá con celo lo establecido en el Código de Infancia y la Adolescencia, especialmente en relación con las responsabilidades de los medios de comunicación, indicadas en el Artículo 47. 
En constancia firma(n) el (los) investigador(es) responsable(s) del proyecto,

- Firma:

Nombre del Investigador responsable:

Rol:

$\mathrm{N}^{\mathrm{o}}$ Identificación:

Fecha: ___ I

- Firma:

Nombre del Investigador responsable:

Rol:

$\mathrm{N}^{\circ}$ Identificación:

Fecha: 11 


\section{Datos sociodemográficos:}

- Nombre y apellido:

- Edad:

- Género/Sexo: Hombre

Mujer

- Fecha de nacimiento:

- Lugar de nacimiento:

- Nivel o estrato económico: No sabe:

- Dirección de vivienda:

- Tipo de vivienda:

- Estado civil:

Soltero: Casado: Divorciado: Viudo: No responde:

- Pertenece a algún grupo étnico:

Si: No: ¿Cuál?:

- Información familiar:

¿Con quién vive? Papá: Mamá: Hermanos: Otros:

Nombres:

- Escolarización:

Primaria: Bachillerato:

- Ejerce actividades diferentes a las académicas:

Si: No: ¿Cuál?:

- Estado de salud:

Bueno:___ No tan bueno:____ Malo: ¿Qué enfermedad o problema de salud padece?:

- Ocupación: 


\section{Examen mental}

\section{1.- Apariencia / Porte:}

- Atuendo:

- Higiene corporal:

- Mirada y expresión:

- Postura:

Observaciones:

\section{2.- Actitud:}

Coopera:

Abierto:

Temeroso:

Hostil:

Observaciones:

\section{3.- Conciencia:}

¿Cual es tu nombre?

¿Que edad tiene?

¿Sabe estamos en este preciso momento?

Me puede decir, ¿Como llega a su casa al salir de acá?

- Se encuentra en estado de vigilia (capacidad de respuesta completa): $\mathrm{Si}$ No

- Presenta un estado de estupor (escasa o nula actividad espontánea): Si No 
- Se encuentra en estado de coma (ausencia total de respuesta) : Si No

Observaciones:

\section{4.- Orientación:}

¿Qué desayuno?

¿Cómo se llega a su casa?

¿Sabe qué fecha es hoy?

- Demuestra orientación autopsíquica (persona): $\mathrm{Si} \_$No

- Demuestra orientación alopsíquica (tiempo-espacio): $\mathrm{Si} \_$No _

Observaciones:

\section{5.- Lenguaje:}

¿Que momento ocurrido en el transcurso de su vida le causa más felicidad? ¿Como sucedió?

- Habla incesantemente: $\mathrm{Si}$ No

- Habla normal: Si No

- Habla escasa: Si

No

Señale con una "X" si la persona presenta al hablar:

Prolijidad

Auto-referencia

Perseveración

Inconsecuencia

Incoherencia Incongruencia

Neologismos

Balbuceo

Tartamudeo

- Volumen del habla: Normal

Alto

Bajo 
Observaciones:

\section{6.- Pensamiento:}

¿Por qué crees que es importante estudiar?

¿Qué proyecciones tienes a futuro?

¿Como piensas llevarlas a cabo?

- Marca con una "X" como es el curso del pensamiento:

Normal Intrusivo Fuga de ideas Tangencial

Circunstancial (detallado)

Disociado

(desconectado)

Taquipsiquia

Bradipsiquia

- Estructura del pensamiento: Lógico

Ilógico

Observaciones:

\section{7.- Atención:}

- Responde a la estimulación visual: Si No

- Responde a la estimulación auditiva: Si No

- Atención dispersa: Si__ No

- Atención selectiva: $\mathrm{Si} \_$No

- Atención sostenida: $\mathrm{Si}$ No

Observaciones: 


\section{8.- Memoria:}

¿Cual era tu actividad favorita cuando más pequeño?

Con anterioridad se le enuncia un dato, que será retomado en este apartado ( Un nombre, una fecha, número, dirección, ect...) con la pregunta de ¿Me puedes recordar lo que te dije hace un momento?

- La persona presenta memoria:

Retrógrada

Anterógrada De retención Largo plazo

Corto plazo

Observaciones:

\section{9.- Afecto:}

¿Cómo te sientes en realizando actividades en el colegio?

- La persona presenta un afecto:

1. Amplio y apropiado (expresiones son de acuerdo a sus emociones)

2. Aplanado (ausencia de cualquier expresión afectiva)

3. Embotado (reducción de la intensidad de la expresión emocional)

4. Restringido (disminución de la intensidad de la expresión emocional)

5. Lábil (cambios repentinos de emoción)

6. Disociado (las expresiones no van de acuerdo a las emociones)

Observaciones:

\section{0.- Sensopercepción:}

¿Alguna vez has visto las cosas u objetos deformados? ¿Has visto cosas que los demás no? En los momentos que estás solo ¿has escuchado voces que te hablan? ¿Has sentido olores 
extraños que otros no perciben? En algún momento ¿ha experimentado cosas raras en tu cuerpo, como si te tocaran no habiendo nadie presente?

- La persona presenta alucinaciones: $\mathrm{Si}{ }_{\text {___ No }}$

En caso de que diga que sí, especifique con una " $\mathrm{X}$ ” cuál:

Olfativas Gustativas Táctiles Visuales Auditivas

Observaciones:

\section{1.- Introspección:}

- Presenta una introspección:
Intelectual
Emocional
Confiabilidad

Observaciones:

\section{2.- Sueño:}

¿Cuantas hora duermes normalmente?

¿Por qué no duermes lo suficiente?

¿Te cuesta conciliar el sueño?

- La persona presenta:

Insomnio Sueño de conciliación

Sueño de mantenimiento

Sueño de despertar Disminución de la necesidad del sueño

Desincronización del ciclo del sueño 
Observaciones:

\section{Motivo de entrevista:}

- ¿Me puedes decir por qué estás aquí/ que hizo que vinieras a terapia? o ¿En qué te gustaría trabajar?

\section{Explicación del proceso:}

Se harán preguntas orientadas al reconocimiento de la problemática presente según el estudio. Mediante una conversación bidireccional directa y profunda se procederá a una recogida datos sujetos por la diversidad de preguntas realizadas al entrevistado, todos con un propósito en contexto, todo con el debido respeto y confidencialidad del consultante.

\section{Preguntas}

\section{- Preguntas para evaluar habilidades sociales en el aula}

1. ¿Cómo sueles afrontar las situaciones complejas como problemas, dificultades o no poder realizar una tarea?

2. ¿Sueles pedir ayuda para resolver estas situaciones? Si es así, ¿De qué forma pides dicha ayuda?

3. Durante la clase, ¿expresas tus opiniones de forma libre y respetuosa, aunque estén en contra del pensamiento de los demás? ¿Como lo haces?

4. ¿Cuál es la manera en la que haces preguntas en clase sobre temas que no entiendes?

5. ¿Prestas atención a la persona que te está hablando y haces un esfuerzo para comprender lo que te está diciendo?

6. Cuando tienes opiniones diferentes a las de alguien ¿Intentas llegar a un acuerdo justo? ¿Qué acciones tomas?

7. ¿De qué manera expresas tus sentimientos a los demás?

8. ¿Usas algún método de organización para realizar una tarea? ¿Qué método?

9. ¿Cómo actúas cuando conoces nuevas personas? 


\section{- Preguntas para evaluar la convivencia}

1. ¿Cómo son las relaciones con tus compañeros y profesores?

2. En el momento de que tus compañeros y tu deban ponerse de acuerdo en algo ¿Cómo lo hacen?

3. ¿Has recibido alguna agresión física o insulto dentro del centro educativo? ¿Cómo la enfrentaste?

4. ¿Puede decirme cuando comenzaron los problemas de convivencia con sus compañeros?

5. ¿Cómo fueron evolucionando sus problemas de convivencia?

6. ¿Cuáles son, en su opinión, las razones de su problema de convivencia?

7. ¿Has intentado tomar acciones que te ayuden a mejorar esta situación? ¿Qué acciones?

8. ¿De qué manera se resuelven los conflictos que se presentan en tu institución educativa?

\section{Intervención con padres de familia o tutores legales}

Durante el proceso de entrevista es necesaria la participación de los padres de familia o tutores, para evidenciar si tenían conocimiento sobre la situación que se esté presentando, para que así aporten a la resolución de los conflictos que presente el estudiante. Tal como se enuncia en el Manual de terapia infantil gestáltica, donde dice Cornejo (1996):

Los padres, los padres de nuestro paciente, son tan importantes como nuestro propio paciente. Primero, porque es parte importante de su mundo y luego, porque si existe una buena alianza entre ellos y nosotros, la mitad de la terapia casi está hecha (p.17)

\section{Intervención con maestros}

Dado que el caso, durante el proceso de entrevista al estudiante mediante los resultados, no se le determina necesario seguir con el mismo, se hará la remisión al docente verificar cual es el propósito de la remisión, tal como formulan Bassedas, Huguet, Marrodan, Oliván, Planas, 
Rossell y Vilella (1998) “captar los aspectos más vivenciales de la relación que establece el maestro con la problemática del niño. Intentar entender las vivencias, las representaciones y los puntos de preocupación que el maestro tiene en relación a la problemática del niño” (p. 82).

\section{Conclusión}

\section{Concepto}

La presente entrevista está dirigida a la investigación y evaluación de las habilidades sociales, siendo estas entendidas según Inés Monjas, las habilidades sociales son las «conductas o destrezas sociales específicas requeridas para ejecutar competentemente una tarea de índole interpersonal. Implica un conjunto de comportamientos adquiridos y aprendidos y no unrasgo de personalidad. Son un conjunto de comportamientos interpersonales complejos que se ponen en juego en la interacción con otras personas» (Rosales, Caparrós \& Molina, 2013); y a su vez se enfoca igualmente en evaluar las condiciones de convivencia que presenta el aula de la Institución educativa, en la dimensión psicoeducativa, el concepto de convivencia refiere un análisis de los sentimientos y emociones que se exigen para relacionarse bien con los demás. Una dimensión social y moral que incluye el reconocimiento del otro, como semejante y diferente al mismo tiempo (Bravo \& Herrera, 2011). 


\section{Referencias:}

Bassedas, E., Huguet, T., Marrodan, M., Oliván, M., Planas, M., Rossell, M., \& Vilella, M. (1998). Intervención educativa y diagnóstico psicopedagógico. Paidós.

Bravo, I. \& Herrera, L. (2011). Convivencia escolar en educación primaria. Las habilidades sociales del alumnado como variable moduladora. Dedica. Revista de Educação e Humanidades. 1. pp 173-212.

Cornejo, L. (1996). Manual de terapia infantil gestáltica. Bilbao: Desclee de Brower.

Heredia, M. C., Santaella, G., \& Somarriba, L. (2012). Informe psicológico. Textos de apoyo didáctico, Facultad de Psicología, UNAM.

Morrison, J. (2015). La entrevista psicológica. Editorial El Manual Moderno.

Perpiñá, C. (2014). Manual de la entrevista psicológica. Ediciones Pirámide.

Rosales, J. J. J., Caparrós, F. B. M. D. M., \& Molina, R. I. (2013). Habilidades sociales.

Universidad Santo Tomás Sede De Vllavicencio Dirección De Investigación E Innovación Comité De Ética, Bioética E Integridad

Científica. (27-01-2020). Formato de Asentimiento informado (Versión 01. No.18) 


\section{Manual del Formato de la entrevista}

Introducción: A continuación, encontrará una serie de afirmaciones que se aplican a través de una entrevista para evaluar las habilidades sociales y la convivencia entre estudiantes de educación primaria y bachillerato.

Tipo de entrevista: Semi-estructurada

Tipo de entrevista según su variación: Entrevista narrativa

Este formato se aplica en caso de que el estudiante sea reportado o se reporte como mala convivencia con sus compañeros.

\section{Asentimiento informado:}

Este proceso es de suma importancia, pues el asentimiento informado consiste en explicarle a la persona cómo se desarrollará el proceso de evaluación, es decir, se le plantea que se tomaran apuntes o grabaciones de voz de lo que se converse, teniendo en cuenta que la información que no quiere que revelemos se mantendrá bajo confidencialidad, más sin embargo se tendrá que realizar informes con las generalidades del motivo de la entrevista; y a su vez se aclara en este mismo punto que cualquier información compartida donde se ponga en riesgo su vida o la de otra persona será comunicado a las autoridades legales; el estudiante si decide firmar el asentimiento, dará luz verde para poder seguir con el proceso

\section{Datos personales o Datos sociodemográficos:}

Estos son importantes porque permiten ampliar la información que se tiene sobre el alumno, permitiendo conocer con quienes vive, su edad, estrato social, etc; el conocimiento de estos datos personales también se presta para analizar si estos tienen que ver con el problema; aparte de que también ayudan a desarrollar el examen mental.

1. Nombre y apellido: El nombre del estudiante no se puede dar por sentado así ya se sepa, que el alumno responda permite evaluar el grado de conciencia.

2. Edad: Saber la edad del alumno permite determinar si su lenguaje, pensamiento, etc; va acorde a su etapa de madurez. 
3. Género/Sexo: Aquí se puede identificar si la persona se siente gusto con su género

4. Lugar y Fecha de nacimiento: Esta pregunta ayuda a ver el grado de orientación y conciencia del estudiante

5. Nivel o estrato económico y tipo de vivienda: En este se puede evaluar el medio que rodea a la persona, es necesario indagar si vive de arriendo, propia, familiar o invasión.

6. Dirección de vivienda: Esta pregunta permite evaluar la orientación del sujeto.

7. Estado civil: Esta pregunta se hace solo a mayores de edad pero pueden haber casos donde adolescentes de 16 años pueden vivir en unión libre o estén casados ya que esta es la edad legal para contraer nupcias con permiso de los padres

8. Pertenece a algún grupo étnico: Permitirá ampliar la información del alumno y tal vez si la respuesta es sí tenga que ver con su problema.

9. Información familiar: Permitirá identificar qué relación lleva con su familia como buena, mala o regular; con quienes convive y al ser menor de edad permitirá saber quién está a cargo de su cuidado.

10. Escolarización: Ayuda a evidenciar el desarrollo que a tenido el estudiante y si es acorde a su edad, se debe tener en cuenta si es un niño o un adulto.

11. Ejerce actividades diferentes a las académicas: Permite evidenciar que tan activo es la persona, dependiendo de su edad.

12. Estado de salud: Hay que saber si el paciente presenta o ha presentadoenfermedades, tanto hereditarias como normales o que hayan sido producidas por algo, este tipo de historial puede ser clave para el proceso a llevar, especialmente qué tipo de enfermedades han sido teniendo en cuenta la edad del paciente.

\section{Examen mental:}

Es una forma de recolección de datos; el examen mental permite explorar y recoger el estado emocional, el funcionamiento y la capacidad mental de una persona, a partir de la observación y exploración ordenada de signos (lo observable, tos, fiebre, etc) y síntomas (lo no observable, dolor de cabeza, estómago, etc). 
Se evalúa a partir de:

* Porte y actitud: Forma de vestir, edad mental, empatía, comportamientos adecuados para la edad.

\section{* Conciencia:}

1. Vigilia: (Estado de alerta normal) Atento a la conversación y responde a tiempo y de forma coherente.

2. Obnubilación: (Somnolencia) Presenta una capacidad de respuesta muy lenta durante la conversación.

3. Estupor: Escasa o nula actividad de espontaneidad.

4. Coma: Ausencia total de respuesta.

* Orientación: La evaluación se hace desde desde la fase inicial de la entrevista se le puede preguntar la fecha o dia en el momento y así se determina su orientación de tiempo o si sabe explicar dónde se encuentra y cómo llegó ahí se evalúa orientación de espacio.

Siendo la autopsíquica la orientación en la cual el sujeto es capaz de identificarse así mismo, es decir es capaz de dar su nombre, apellidos, fecha de nacimiento, etc. Y la alopsíquica la forma en la que se orienta en tiempo-espacio (incluye tanto la orientación temporal, o capacidad para conocer el momento (año, mes, día) en que se vive realmente, la espacial o capacidad para conocer el lugar (ciudad, calle, edificio) en que la persona se encuentra, etc)

Lenguaje: La forma en que se expresa el estudiante al hablar teniendo en cuenta el vocabulario y léxico.

* Pensamiento: Se evalúa para evidenciar la rapidez de pensamiento del alumno; mientras se habla con él se le realizarán preguntas para determinar si presenta bradipsiquia (pensamiento lento) o ideofugable (pensamiento rápido); también se puede evidenciar si el paciente presenta alucinaciones, delirios o ideas obsesivas.

- Atención: Este dará a conocer si el estudiante mantiene el foco atencional en una actividad por largo tiempo, o si tiende a distraerse con facilidad 
* Memoria:

1. Memoria a corto plazo: Capacidad para mantener en mente de forma activa una pequeña cantidad de información, de forma que se encuentre disponible durante un corto periodo de tiempo

2. Memoria a largo plazo: Capacidad del cerebro para almacenar hechos, conocimientos e información por un largo tiempo

3. Memoria retrógrada: Trata de no recordar el pasado.

4. Memoria anterógrada: No se puede aprender o retener información nueva

Afecto: Estado emocional del estudiante a la hora de expresarse o contar sucesos; se puede presentar contradicción entre el contexto de una historia y el afecto del paciente, es decir, estar contando algo trágico, pero reír mientras lo cuenta o viceversa.

* Sensopercepción: La relación que se da entre las sensaciones y la percepción de esta, es decir, la forma en que por medio de sus 5 sentidos percibe o entiende lo que lo rodea.

* Introspección: La capacidad de hacer una autoevaluación o reflexión sobre sí mismo.

* Sueño: Determinar si la persona presenta horas de sueño adecuadas, pues el estar bien descansado permitirá mayor atención y capacidad para responder o expresarse que si lleva varios días sin dormir bien.

\section{Motivo de consulta:}

Es necesario saber las razones por las que el estudiante está en terapia para desarrollar un plan de intervención, como un proceso de relación interpersonal.

(Si el estudiante sabe las razones se comienza el proceso, si no sabe se le explica, puede ser por que ha tenido malos comportamientos en clase, al relacionarse con sus compañeros o hasta con sus profesores). 
Preguntas que se harán en la entrevista

. Preguntas para evaluar habilidades sociales en el aula:

1. ¿Cómo sueles afrontar las situaciones complejas como problemas, dificultades o no poder realizar una tarea? Con esto se busca evidenciar de qué manera el individuo es capaz de confrontar sus propios dilemas, y si los identifica.

2. ¿Sueles pedir ayuda para resolver estas situaciones? Si es así, ¿De qué forma pides dicha ayuda? Siendo esta pregunta complementaria a la anterior, se busca ahondar en el tema del afrontamiento y resolución de los problemas identificando si el alumno y si los identifica de buscar apoyo en alguien más.

3. Durante la clase, ¿expresas tus opiniones de forma libre y respetuosa, aunque estén en contra del pensamiento de los demás? Se refleja si el estudiante se ve presionado por los juicios que hacen sus compañeros, frente a su libertad de pensamiento, evitando la influencia de la opinión de los otros.

4. ¿Cual es la manera en la que haces preguntas en clase sobre temas que no entiendes? La retroalimentación de los saberes no aprendidos, se puede ver reprimida por el hecho de tener posibilidades a ser juzgado por sus compañeros y las opiniones que tengan acerca del valor de la cuestión.

5. ¿Prestas atención a la persona que te está hablando y haces un esfuerzo para comprender lo que te está diciendo? Con esto se pretende evaluar la habilidad social de la escucha.

6. Cuando tienes opiniones diferentes a las de alguien iIntentas llegar a un acuerdo justo? Podemos observar si esta persona puede hacer frente a estas situaciones sociales dependiendo de la alta compresión y empatía que tenga hacia los otros.

7. ¿De qué manera expresas tus sentimientos a los demás? La comunicación de los sentimientos del entrevistado hace presente la conciencia que tiene de los mismo, y que tipo de relación puede establecer para facilitar este proceso. 
8. ¿Usas algún método de organización para realizar una tarea? Apreciamos si el entrevistado emplea de manera sistemática un orden según la funcionalidad de las cosas y el valor que perciba el mismo.

9. ¿Cómo actúas cuando conoces nuevas personas? La personalidad progresa a la par con la sociabilidad, y de tal manera se pondrá en juicio el desempeño en la capacidad al interactuar, y la comparación entre pares.

\section{- Preguntas para evaluar la convivencia:}

1. ¿Cómo son las relaciones con tus compañeros y profesores? Permite evidenciar si se presentan problemas a la hora de relacionarse; si hay problemas se podría preguntar el porqué de estos, y que diferencias entre ellos causan la desigualdad; así como puede ser relaciones positivas y amigables.

2. En el momento de que tus compañeros y tu deban ponerse de acuerdo en algo ¿Cómo lo hacen? Con esta pregunta evaluaremos la capacidad de negociación del alumno como una alternativa a la resolución de dilemas de forma violenta.

3. ¿Has recibido alguna agresión física o insulto dentro del centro educativo? Que se presenten agresiones físicas o verbales entre compañeros puede ser una gran evidencia de diferencias entre ellos y por ende estas faltas de respeto generan mal ambiente escolar, afectando la convivencia.

4. ¿Puede decirme cuando comenzaron los problemas de convivencia con sus compañeros?

5. ¿Sabe cómo fueron evolucionando sus problemas de convivencia?

- Estas dos preguntas ayudan a especificar la duración y raíz de los problemas; teniendo esto presente se puede empezar a desarrollar u plan de mejoramiento de la convivencia.

6. ¿Cuáles son, en su opinión, las razones de su problema de convivencia? Es importante indagar sobre la opinión y como el alumno percibe la situación por la que pasa. 


\section{7. ¿Has intentado tomar acciones que te ayuden a mejorar esta situación? Se hace Esta} pregunta con la intención de conocer las alternativas que el alumno considera pertinente para resolver el problema de convivencia.

\section{8. ¿De qué manera se resuelven los conflictos que se presentan en tu institución educativa?}

Saber cómo los estudiantes perciben las razones de sus problemas; cómo suelen solucionar sus diferencias o de qué forma han intentado solucionarlos teniendo en cuenta lo que es correcto y no como dialogar o llegar a acuerdos permite evidenciar que tanto interés tienen los estudiantes por mejorar la convivencia, pero también se puede dar que nunca hayan intentado soluciones pacíficas y hasta puede que nunca se lo hayan planteado y elijan siempre solucionar todo con agresiones y aun así no tengan total claridad de qué es lo que genera los conflictos.

\section{Intervención con padres de familia o tutores legales}

Los padres son parte fundamental en el proceso de entrevista con el alumno, ya que son la base del proceso al que se dará inició. La mayoría de veces los padres no tienen conocimiento de los hechos que le ocurren a su hijo, por lo tanto, es la institución quien se encarga de realizarles una citación para contextualizarlos, y solicitar de su ayuda para la aplicación de las estrategias tomadas por el psicólogo educativo.

\section{Intervención con maestros}

Los maestros no solo están encargados de impartir materias, también deben de estar al pendiente de las situaciones que les sucedan a sus alumnos, por lo tanto, si se evidencia alguna manifestación que requiera de la intervención del psicólogo educativo, es su obligación informar al mismo, para dar inicio al proceso de acompañamiento psicológico, para poder dar solución a los conflictos que se presenten.

\section{Conclusión}

Se realiza el cierre de la entrevista, se debe hacer sentir al entrevistado que valió el tiempo de participación en la misma, mas no que vago todo lo que expresó al entrevistador, si es necesaria nuevamente agendar otra sesión, se comunicarán la información necesaria, tal como fecha, hora y lugar. 
Por otro lado, es de suma importancia hacer la trascripción de manera inmediata de toda la información recolectada en la entrevista, para realizar un informe más completo, alejado de distorsiones, que puedan afectar el proceso, además debe de ser claro para que, si dado el caso se necesite retomar la información postulada, pueda ser de fácil interpretación. Así mismo, si se realizó alguna grabación ya sea de audio o video, sea adjuntaba previamente. 